\title{
Analytical Solution of Heat Conduction for Hollow Cylinders with Time-Dependent Boundary Condition and Time-Dependent Heat Transfer Coefficient
}

\author{
Te-Wen $\mathrm{Tu}^{1}$ and Sen-Yung Lee ${ }^{2}$ \\ ${ }^{1}$ Department of Mechanical Engineering, Air Force Institute of Technology, No. 198 Jieshou W. Road, Gangshan Township, \\ Kaohsiung 820, Taiwan \\ ${ }^{2}$ Department of Mechanical Engineering, National Cheng Kung University, No. 1 University Road, Tainan 701, Taiwan
}

Correspondence should be addressed to Sen-Yung Lee; sylee@mail.ncku.edu.tw

Received 5 May 2015; Accepted 21 July 2015

Academic Editor: Assunta Andreozzi

Copyright (C) 2015 T.-W. Tu and S.-Y. Lee. This is an open access article distributed under the Creative Commons Attribution License, which permits unrestricted use, distribution, and reproduction in any medium, provided the original work is properly cited.

\begin{abstract}
An analytical solution for the heat transfer in hollow cylinders with time-dependent boundary condition and time-dependent heat transfer coefficient at different surfaces is developed for the first time. The methodology is an extension of the shifting function method. By dividing the Biot function into a constant plus a function and introducing two specially chosen shifting functions, the system is transformed into a partial differential equation with homogenous boundary conditions only. The transformed system is thus solved by series expansion theorem. Limiting cases of the solution are studied and numerical results are compared with those in the literature. The convergence rate of the present solution is fast and the analytical solution is simple and accurate. Also, the influence of physical parameters on the temperature distribution of a hollow cylinder along the radial direction is investigated.
\end{abstract}

\section{Introduction}

The problems of transient heat flow in hollow cylinders are important in many engineering applications. Heat exchanger tubes, solidification of metal tube casting, cannon barrels, time variation heating on walls of circular structure, and heat treatment on hollow cylinders are typical examples. It is well known that if the temperature and/or the heat flux are prescribed at the boundary surface, then the heat transfer system includes heat conduction coefficient only; on the other hand, if the boundary surface dissipates heat by convection on the basis of Newton's law of cooling, the heat transfer coefficient will be included in the boundary term.

For the problem of heat conduction in hollow cylinders with time-dependent boundary conditions of any kinds at inner and outer surfaces, the associated governing differential equation is a second-order Bessel differential equation with constant coefficients. After conducting a Hankel transformation, the analytical solutions can be obtained and found in the textbook by Ozisik [1].
For the heat transfer in hollow cylinders with mixed type boundary condition and time-dependent heat transfer coefficient simultaneously, the problem cannot be solved by any analytical methods, such as the method of separation of variable, Laplace transform, and Hankel transform. Few studies in Cartesian coordinate system can be found and various approximated and numerical methods were proposed. By introducing a new variable, Ivanov and Salomatov $[2,3]$ together with Postol'nik [4] transformed the linear governing equation into a nonlinear form. After ignoring the nonlinear term, they developed an approximated solution, which was claimed to be valid for the system with Biot number being less than 0.25. Moreover, Kozlov [5] used Laplace transformation to study the problems with Biot function in a rational combination of sines, cosines, polynomials, and exponentials. Even though it is possible to obtain the exact series solution of a specified transformed system, the problem is the computation of the inverse Laplace transformation, which generally requires integration in the complex plane. Becker et al. [6] took finite difference method and Laplace 
transformation method to study the heating of the rock adjacent to water flowing through a crevice. Recently, Chen and his colleagues [7] proposed an analytical solution by using the shifting function method for the heat conduction in a slab with time-dependent heat transfer coefficient at one end. Yatskiv et al. [8] studied the thermostressed state of cylinder with thin near-surface layer having time-dependent thermophysical properties. They reduced the problem to an integrodifferential equation with variable coefficients and solved it by the spline approximation.

In addition, different approximation methods such as the iterative perturbation method [9], the time-varying eigenfunction expansion method with finite integral transforms $[10,11]$, generalized integral transforms [12], and the Lie point symmetry analysis [13] were used to study this kind of problems. Various inverse schemes for determining the timedependent heat transfer coefficient were developed by some researchers [14-20].

According to the literature, because of the complexity and difficulty of the methodology, none of any analytical solutions for the heat conduction in a hollow cylinder with time-dependent boundary condition and time-dependent heat transfer coefficient existed. This work extends the methodology of shifting function method $[7,21,22]$ to develop an analytical solution with closed form for the heat transfer in hollow cylinders with time-dependent boundary condition and time-dependent heat transfer coefficient simultaneously. By setting the Biot function in a particular form and introducing two specially chosen shifting functions, the system is transformed into a partial differential equation with homogenous boundary conditions and can be solved by series expansion theorem. Examples are given to demonstrate the methodology and numerical results are compared with those in the literature. And last but not least, the influence of physical parameters on the temperature profile is studied.

\section{Mathematical Modeling}

Consider the transient heat conduction in heat exchanger tubes as shown in Figure 1. A fluid with time-varying temperature is running inside the hollow cylinder and the heat is dissipated by the time-dependent convection at the outer surface into an environment of zero temperature. The governing differential equation of the system is

$$
\frac{\partial^{2} T}{\partial r^{2}}+\frac{1}{r} \frac{\partial T}{\partial r}=\frac{1}{\alpha} \frac{\partial T}{\partial t}, \quad a<r<b, t>0,
$$

where $T$ is the temperature, $r$ is the space variable, $\alpha$ is the thermal diffusivity, $t$ is the time variable, and $a$ and $b$ denote inner and outer radii, respectively. The boundary and initial conditions of the boundary value problem are

$$
\begin{array}{cc}
T=H(t), \quad \text { at } r=a, \\
-k \frac{\partial T}{\partial r}=h(t) T, \quad \text { at } r=b, \\
T(r)=T_{0}(r), \quad \text { when } t=0 .
\end{array}
$$

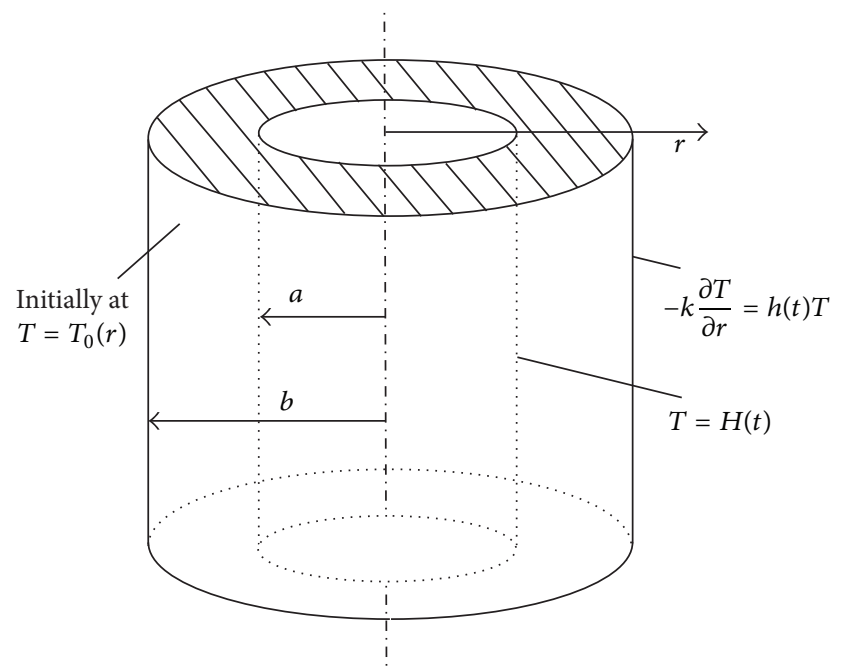

Figure 1: Hollow cylinders with time-dependent temperature and time-dependent heat transfer coefficient at inner and outer surfaces.

Here, $H(t)$ is a time-dependent temperature function at the inner surface, $k$ is the thermal conductivity, $h(t)$ is a timedependent heat transfer coefficient function, and $T_{0}(r)$ is an initial temperature function. For consistence in initial temperature field, $H(0)$ must be equal to $T_{0}(a)$. The above problem can be normalized by defining

$$
\begin{aligned}
\theta & =\frac{T}{T_{r}}, \\
R & =\frac{r}{b}, \\
\tau & =\frac{\alpha t}{b^{2}}, \\
\bar{r} & =\frac{a}{b}, \\
\psi(\tau) & =\frac{H(t)}{T_{r}}, \\
\operatorname{Bi}(\tau) & =\frac{h(t) b}{k}, \\
\theta_{0}(R) & =\frac{T_{0}(r)}{T_{r}},
\end{aligned}
$$

where $T_{r}$ is a constant reference temperature, and the dimensionless boundary value problem will then become

$$
\begin{aligned}
\frac{\partial^{2} \theta}{\partial R^{2}}+\frac{1}{R} \frac{\partial \theta}{\partial R} & =\frac{\partial \theta}{\partial \tau}, \quad \bar{r}<R<1, \tau>0, \\
\theta & =\psi(\tau), \quad \text { at } R=\bar{r}, \\
\frac{\partial \theta}{\partial R}+\operatorname{Bi}(\tau) \theta & =0, \quad \text { at } R=1, \\
\theta(R, 0) & =\theta_{0}(R), \quad \text { when } \tau=0 .
\end{aligned}
$$


To keep the boundary condition of the third kind at outer surface in the following analysis, one sets the Biot function $\operatorname{Bi}(\tau)$ in the form of

$$
\operatorname{Bi}(\tau)=\delta+F(\tau),
$$

where $\delta$ and $F(\tau)$ are defined as

$$
\begin{aligned}
\delta & =\operatorname{Bi}(0), \\
F(\tau) & =\operatorname{Bi}(\tau)-\operatorname{Bi}(0) .
\end{aligned}
$$

It is obvious that $F(0)=0$, and the boundary condition at $R=1$ can be rewritten as

$$
\frac{\partial \theta}{\partial R}+\delta \theta=-F(\tau) \theta, \quad \text { at } R=1
$$

\section{The Shifting Function Method}

3.1. Change of Variable. To find the solution for the secondorder differential equation with time-dependent boundary condition and time-dependent heat transfer coefficient at different surfaces, the shifting function method $[7,21,22]$ was extended by taking

$$
\theta(R, \tau)=v(R, \tau)+g_{1}(R) f_{1}(\tau)+g_{2}(R) f_{2}(\tau),
$$

where $v(R, \tau)$ is called transformed function, $g_{i}(R)(i=1,2)$ are two shifting functions to be specified, and $f_{i}(\tau)(i=1,2)$ are the auxiliary time functions defined as

$$
\begin{aligned}
& f_{1}(\tau)=\psi(\tau), \\
& f_{2}(\tau)=-F(\tau) \theta(1, \tau) .
\end{aligned}
$$

Substituting (11) into (4), (5), (10), and (7), one has the following equation:

$$
\begin{aligned}
\frac{\partial^{2} v(R, \tau)}{\partial R^{2}}+\frac{1}{R} \frac{\partial v(R, \tau)}{\partial R} & \frac{\partial v(R, \tau)}{\partial \tau}+g_{1}(R) \frac{d f_{1}(\tau)}{d \tau} \\
= & \left.\frac{d^{2} g_{1}(R)}{d R^{2}}+\frac{1}{R} \frac{d g_{1}(R)}{d R}\right] f_{1}(\tau) \\
- & {\left[\frac{d f_{2}(\tau)}{d \tau}\right.} \\
+ & \left.g_{2}(R) \frac{d^{2} g_{2}(R)}{d R^{2}}+\frac{1}{R} \frac{d g_{2}(R)}{d R}\right] f_{2}(\tau),
\end{aligned}
$$

and the associated boundary and initial conditions now are

$$
\begin{aligned}
& v(\bar{r}, \tau)+g_{1}(\bar{r}) f_{1}(\tau)+g_{2}(\bar{r}) f_{2}(\tau)=f_{1}(\tau), \\
& \text { at } R=\bar{r} \text {, } \\
& \frac{\partial v(1, \tau)}{\partial R}+\delta v(1, \tau)+\left[\frac{d g_{1}(1)}{d R}+\delta g_{1}(1)\right] f_{1}(\tau) \\
& +\left[\frac{d g_{2}(1)}{d R}+\delta g_{2}(1)\right] f_{2}(\tau)=f_{2}(\tau), \quad \text { at } R=1, \\
& v(R, 0)+g_{1}(R) f_{1}(0)+g_{2}(R) f_{2}(0)=\theta_{0}(R) \text {. }
\end{aligned}
$$

Something worthy to mention is that (13) contains three functions, that is, $v(R, \tau)$ and $g_{i}(R)(i=1,2)$, and hence it cannot be solved directly.

3.2. The Shifting Functions. For convenience in the analysis, the two shifting functions are specifically chosen in order to satisfy the following conditions:

$$
\begin{aligned}
& g_{1}(\bar{r})=1, \\
& g_{2}(\bar{r})=0,
\end{aligned}
$$

at $R=\bar{r}$

$$
\begin{aligned}
g_{1}(1) & =0, \\
g_{2}(1) & =0, \\
\frac{d g_{1}(1)}{d R} & =0, \\
\frac{d g_{2}(1)}{d R} & =1,
\end{aligned}
$$

at $R=1$.

Consequently, the shifting functions can be easily determined as

$$
\begin{aligned}
& g_{1}(R)=\left(\frac{1-R}{1-\bar{r}}\right)^{2}, \\
& g_{2}(R)=\frac{(R-1)(R-\bar{r})}{1-\bar{r}} .
\end{aligned}
$$

Substituting these shifting functions and auxiliary time functions into (11) yields

$$
\begin{aligned}
\theta(R, \tau)= & v(R, \tau)+\left(\frac{1-R}{1-\bar{r}}\right)^{2} \psi(\tau) \\
& -\left[\frac{(R-1)(R-\bar{r})}{1-\bar{r}}\right] F(\tau) \theta(1, \tau) .
\end{aligned}
$$

When setting $R=1$ in the equation above, one has the relation

$$
\theta(1, \tau)=v(1, \tau)
$$

Therefore, two functions in governing differential equation (13) are integrated to one. With (16) and (18), (13) can be rewritten in terms of the function $v(R, \tau)$ as

$$
\begin{aligned}
& \frac{\partial v(R, \tau)}{\partial \tau}-\frac{\partial^{2} v(R, \tau)}{\partial R^{2}}-\frac{1}{R} \frac{\partial v(R, \tau)}{\partial R} \\
& \quad-g_{2}(R) \frac{d}{d \tau}[F(\tau) v(1, \tau)]+d_{2}(R) F(\tau) v(1, \tau) \\
& =-g_{1}(R) \dot{\psi}(\tau)+d_{1}(R) \psi(\tau),
\end{aligned}
$$

where $d_{i}(R),(i=1,2)$ are defined as

$$
\begin{aligned}
& d_{1}(R)=\frac{4 R-2}{R(1-\bar{r})^{2}}, \\
& d_{2}(R)=\frac{4 R-(1+\bar{r})}{R(1-\bar{r})} .
\end{aligned}
$$


Meanwhile, the associated boundary conditions of the transformed function turn to homogeneous ones as follows:

$$
\begin{aligned}
v(\bar{r}, \tau) & =0 \\
\frac{\partial v(1, \tau)}{\partial R}+\delta v(1, \tau) & =0 .
\end{aligned}
$$

Since $f_{2}(0)=-F(0) \theta(1,0)$ and $F(0)=0$, hence, the associated initial condition can be simplified as

$$
v(R, 0)=\theta_{0}(R)-g_{1}(R) \psi(0)=v_{0}(R) .
$$

3.3. Series Expansion. To find the solution for the boundary value problem of heat conduction, that is, (19)-(22), one uses the method of series expansion with try functions:

$$
\phi_{n}(R)=J_{0}\left(\lambda_{n} R\right)-\frac{J_{0}\left(\lambda_{n} \bar{r}\right)}{Y_{0}\left(\lambda_{n} \bar{r}\right)} Y_{0}\left(\lambda_{n} R\right)
$$

$$
n=1,2,3, \ldots,
$$

satisfying the boundary conditions (21). Here the characteristic values $\lambda_{n}$ are the roots of the transcendental equation

$$
\frac{J_{0}\left(\lambda_{n} \bar{r}\right) Y_{1}\left(\lambda_{n}\right)-J_{1}\left(\lambda_{n}\right) Y_{0}\left(\lambda_{n} \bar{r}\right)}{J_{0}\left(\lambda_{n} \bar{r}\right) Y_{0}\left(\lambda_{n}\right)-J_{0}\left(\lambda_{n}\right) Y_{0}\left(\lambda_{n} \bar{r}\right)}=\frac{\delta}{\lambda_{n}} .
$$

The try functions have the following orthogonal property:

$$
\int_{\bar{r}}^{1} R \phi_{m}(R) \phi_{n}(R) d R= \begin{cases}0, & m \neq n \\ N_{n}, & m=n,\end{cases}
$$

where the norms $N_{n}$ are

$$
N_{n}=\frac{1}{2 \lambda_{n}^{2}}\left[\left(\lambda_{n}^{2}+\delta^{2}\right) \phi_{n}^{2}(1)-\frac{4}{\pi^{2} Y_{0}^{2}\left(\lambda_{n} \bar{r}\right)}\right] .
$$

Now, one can assume that the solution of the physical problem takes the form of

$$
v(R, \tau)=\sum_{n=1}^{\infty} \phi_{n}(R) q_{n}(\tau), \quad n=1,2,3, \ldots
$$

where $q_{n}(\tau)(n=1,2,3, \ldots)$ are time-dependent generalized coordinates. Substituting solution from (27) into differential equation (19) leads to

$$
\begin{aligned}
& \sum_{n=1}^{\infty}\left\{\phi_{n}(R) \dot{q}_{n}(\tau)-\phi_{n}^{\prime \prime}(R) q_{n}(\tau)-\frac{1}{R} \phi_{n}^{\prime}(R) q_{n}(\tau)\right. \\
& \quad-g_{2}(R) \phi_{n}(1)\left[\dot{F}(\tau) q_{n}(\tau)+F(\tau) \dot{q}_{n}(\tau)\right] \\
& \left.\quad+d_{2}(R) \phi_{n}(1) F(\tau) q_{n}(\tau)\right\}=-g_{1}(R) \dot{\psi}(\tau) \\
& \quad+d_{1}(R) \psi(\tau) .
\end{aligned}
$$

Expanding $g_{1}(R)$ and $d_{1}(R)$ on the right hand side of (28) in series forms we obtain

$$
\begin{aligned}
& \sum_{n=1}^{\infty}\left\{\phi_{n}(R) \dot{q}_{n}(\tau)-\left[\phi_{n}^{\prime \prime}(R)+\frac{1}{R} \phi_{n}^{\prime}(R)\right] q_{n}(\tau)\right. \\
& \quad-g_{2}(R) \phi_{n}(1)\left[F(\tau) \dot{q}_{n}(\tau)+\dot{F}(\tau) q_{n}(\tau)\right] \\
& \left.\quad+d_{2}(R) \phi_{n}(1) F(\tau) q_{n}(\tau)\right\}=\sum_{n=1}^{\infty}\left[-\gamma_{n} \phi_{n}(R) \dot{\psi}(\tau)\right. \\
& \left.\quad+\bar{\gamma}_{n} \phi_{n}(R) \psi(\tau)\right],
\end{aligned}
$$

where $\gamma_{n}$ and $\bar{\gamma}_{n}$ are

$$
\begin{aligned}
\gamma_{n} & =\frac{\int_{\bar{r}}^{1} R \phi_{n}(R) g_{1}(R) d R}{N_{n}}=\frac{1}{(1-\bar{r})^{2}}\left[\xi_{J 1}-2 \xi_{J 2}\right. \\
& \left.+\xi_{J 3}-\frac{J_{0}\left(\lambda_{n} \bar{r}\right)}{Y_{0}\left(\lambda_{n} \bar{r}\right)}\left(\xi_{Y 1}-2 \xi_{Y 2}+\xi_{Y 3}\right)\right] \\
\bar{\gamma}_{n} & =\frac{\int_{\bar{r}}^{1} R \phi_{n}(R) d_{1}(R) d R}{N_{n}}=\frac{2}{(1-\bar{r})^{2}}\left[2 \xi_{J 1}-\xi_{J 0}\right. \\
& \left.-\frac{J_{0}\left(\lambda_{n} \bar{r}\right)}{Y_{0}\left(\lambda_{n} \bar{r}\right)}\left(2 \xi_{Y 1}-\xi_{Y 0}\right)\right]
\end{aligned}
$$

in which $\xi_{W i}(i=0,1,2,3 ; W=J, Y)$ are given as

$$
\begin{aligned}
\xi_{W 0} & =\frac{\int_{\bar{r}}^{1} W_{0}\left(\lambda_{n} R\right) d R}{N_{n}}, \\
\xi_{W 2} & =\frac{\int_{\bar{r}}^{1} R^{2} W_{0}\left(\lambda_{n} R\right) d R}{N_{n}}, \\
\xi_{W 1} & =\frac{\int_{\bar{r}}^{1} R W_{0}\left(\lambda_{n} R\right) d R}{N_{n}}=\frac{1}{\lambda_{n} N_{n}}\left[W_{1}\left(\lambda_{n}\right)\right. \\
- & \left.\bar{r} W_{1}\left(\lambda_{n} \bar{r}\right)\right] \\
\xi_{W 3} & =\frac{\int_{\bar{r}}^{1} R^{3} W_{0}\left(\lambda_{n} R\right) d R}{N_{n}}=\frac{1}{\lambda_{n}^{3} N_{n}}\left[2 \lambda_{n} W_{0}\left(\lambda_{n}\right)\right. \\
+ & \left(\lambda_{n}^{2}-4\right) W_{1}\left(\lambda_{n}\right)-2 \lambda_{n} \bar{r}^{2} W_{0}\left(\lambda_{n} \bar{r}\right) \\
- & \left.\bar{r}\left(\lambda_{n}^{2} \bar{r}^{2}-4\right) W_{1}\left(\lambda_{n} \bar{r}\right)\right] .
\end{aligned}
$$

From (29), one can let

$$
\begin{aligned}
\phi_{n}(R) & \dot{q}_{n}(\tau)-\left[\phi_{n}^{\prime \prime}(R)+\frac{1}{R} \phi_{n}^{\prime}(R)\right] q_{n}(\tau) \\
& -g_{2}(R) \phi_{n}(1)\left[F(\tau) \dot{q}_{n}(\tau)+\dot{F}(\tau) q_{n}(\tau)\right] \\
& +d_{2}(R) \phi_{n}(1) F(\tau) q_{n}(\tau) \\
= & -\gamma_{n} \phi_{n}(R) \dot{\psi}(\tau)+\bar{\gamma}_{n} \phi_{n}(R) \psi(\tau) .
\end{aligned}
$$


After taking the inner product with try function $R \phi_{n}(R)$ and integrating from $\bar{r}$ to 1 , the resulting differential equation now is

$$
\dot{q}_{n}(\tau)+\frac{\lambda_{n}^{2}-\beta_{n} \dot{F}(\tau)+\bar{\beta}_{n} F(\tau)}{1-\beta_{n} F(\tau)} q_{n}(\tau)=\xi_{n}(\tau),
$$

where $\beta_{n}$ and $\bar{\beta}_{n}$ are

$$
\begin{aligned}
\beta_{n} & =\phi_{n}(1) \frac{\int_{\bar{r}}^{1} R \phi_{n}(R) g_{2}(R) d R}{N_{n}}=\frac{\phi_{n}(1)}{1-\bar{r}}\left\{\xi_{J 3}\right. \\
& -(1+\bar{r}) \xi_{J 2}+\bar{r} \xi_{J 1} \\
& \left.-\frac{J_{0}\left(\lambda_{n} \bar{r}\right)}{Y_{0}\left(\lambda_{n} \bar{r}\right)}\left[\xi_{Y 3}-(1+\bar{r}) \xi_{Y 2}+\bar{r} \xi_{Y 1}\right]\right\}, \\
\bar{\beta}_{n} & =\phi_{n}(1) \frac{\int_{\bar{r}}^{1} R \phi_{n}(R) d_{2}(R) d R}{N_{n}}=\frac{\phi_{n}(1)}{1-\bar{r}}\left\{4 \xi_{J 1}\right. \\
& \left.-(1+\bar{r}) \xi_{J 0}-\frac{J_{0}\left(\lambda_{n} \bar{r}\right)}{Y_{0}\left(\lambda_{n} \bar{r}\right)}\left[4 \xi_{Y 1}-(1+\bar{r}) \xi_{Y 0}\right]\right\},
\end{aligned}
$$

and $\xi_{n}(\tau)$ is

$$
\xi_{n}(\tau)=\frac{-\gamma_{n} \dot{\psi}(\tau)+\bar{\gamma}_{n} \psi(\tau)}{1-\beta_{n} F(\tau)} .
$$

The associated initial condition is

$$
q_{n}(0)=\frac{\int_{\vec{r}}^{1} R \phi_{n}(R) v_{0}(R) d R}{N_{n}} .
$$

As a result, the complete solution of the ordinary differential equation (33) subject to the initial condition (36) is

$$
q_{n}(\tau)=Q_{n}(\tau)\left[q_{n}(0)+\int_{0}^{\tau} \frac{\xi_{n}(\varphi)}{Q_{n}(\varphi)} d \varphi\right],
$$

where $Q_{n}(\tau)$ is

$$
Q_{n}(\tau)=e^{-\int_{0}^{\tau}\left(\left(\lambda_{n}^{2}-\beta_{n} \dot{F}(\zeta)+\bar{\beta}_{n} F(\zeta)\right) /\left(1-\beta_{n} F(\zeta)\right)\right) d \zeta} .
$$

After substituting (16), (18), (23), and (27) back to (11), one obtains the analytical solution of the physical problem

$$
\begin{aligned}
\theta(R, \tau)= & \sum_{n=1}^{\infty}\left\{\left[\phi_{n}(R)-\phi_{n}(1) g_{2}(R) F(\tau)\right] q_{n}(\tau)\right\} \\
& +g_{1}(R) \psi(\tau),
\end{aligned}
$$

where the summation is taken over all eigenvalues $\lambda_{n}$ of the problem.

3.4. Constant Heat Transfer Coefficient at $R=1$. When the heat transfer coefficient $h$ at $R=1$ is time-independent, the Biot function is a constant $\delta$ and $F(\tau)=0$. The infinite series solution, (39), is reduced to

$$
\theta(R, \tau)=\sum_{n=1}^{\infty}\left\{\phi_{n}(R) q_{n}(\tau)+\gamma_{n} \phi_{n}(R) \psi(\tau)\right\},
$$

where the generalized coordinates $q_{n}(\tau)$ are

$$
\begin{gathered}
q_{n}(\tau)=e^{-\lambda_{n}^{2} \tau}\left\{q_{n}(0)+\int_{0}^{\tau}\left[-\gamma_{n} \dot{\psi}(\varphi)+\bar{\gamma}_{n} \psi(\varphi)\right]\right. \\
\left.\cdot e^{\lambda_{n}^{2} \varphi} d \varphi\right\} .
\end{gathered}
$$

The $q_{n}(0)$ 's for the problem under consideration are

$$
q_{n}(0)=\frac{\int_{\vec{r}}^{1} R \phi_{n}(R)\left[\theta_{0}(R)-\psi(0) g_{1}(R)\right] d R}{N_{n}} .
$$

Introducing (42) in (41) and performing integration by parts, we can get

$$
\begin{aligned}
& q_{n}(\tau)=\frac{e^{-\lambda_{n}^{2} \tau}}{N_{n}}\left[\int_{\bar{r}}^{1} R \phi_{n}(R) \theta_{0}(R) d R\right. \\
& \left.-\frac{2}{\pi Y_{0}\left(\lambda_{n} \bar{r}\right)} \int_{0}^{\tau} e^{\lambda_{n}^{2} \varphi} \psi(\varphi) d \varphi\right]-\gamma_{n} \psi(\tau) .
\end{aligned}
$$

Substituting (43) into (40) yields the temperature distribution:

$$
\begin{gathered}
\theta(R, \tau)=\sum_{n=1}^{\infty} \frac{e^{-\lambda_{n}^{2} \tau}}{N_{n}} \phi_{n}(R)\left[\int_{\bar{r}}^{1} R \phi_{n}(R) \theta_{0}(R) d R\right. \\
\left.-\frac{2}{\pi Y_{0}\left(\lambda_{n} \bar{r}\right)} \int_{0}^{\tau} e^{\lambda_{n}^{2} \varphi} \psi(\varphi) d \varphi\right] .
\end{gathered}
$$

This solution is the same as that obtained via the integral transform method by Özisik [1].

\section{Verification and Example}

To illustrate the previous analysis and the accuracy of the three-term approximation solution, one examines the following case.

The time-dependent boundary condition $\psi(\tau)$ considered at $R=\bar{r}$ is taken as

$$
\psi(\tau)=a_{1}-b_{1} e^{-s_{1} \tau} \cos \omega_{1} \tau,
$$

and differentiating it with respect to $\tau$ leads to

$$
\dot{\psi}(\tau)=b_{1} e^{-s_{1} \tau}\left(s_{1} \cos \omega_{1} \tau+\omega_{1} \sin \omega_{1} \tau\right),
$$

where $a_{1}$ and $b_{1}$ are two arbitrary constants and $s_{1}$ and $\omega_{1}$ are two parameters.

The Biot function considered at boundary $R=1$ is

$$
\operatorname{Bi}(\tau)=a_{2}-b_{2} e^{-s_{2} \tau} \cos \omega_{2} \tau,
$$

where $a_{2}$ and $b_{2}$ are two arbitrary constants and $s_{2}$ and $\omega_{2}$ are two parameters. According to (8)-(9), we obtain

$$
\begin{aligned}
\delta & =a_{2}-b_{2}, \\
F(\tau) & =b_{2}\left(1-e^{-s_{2} \tau} \cos \omega_{2} \tau\right), \\
\dot{F}(\tau) & =b_{2} e^{-s_{2} \tau}\left(s_{2} \cos \omega_{2} \tau+\omega_{2} \sin \omega_{2} \tau\right) .
\end{aligned}
$$


Consequently, the temperature distribution in the hollow cylinder is

$$
\begin{aligned}
& \theta(R, \tau)=\sum_{n=1}^{\infty}\left\{q _ { n } ( \tau ) \left[\phi_{n}(R)\right.\right. \\
& \left.\left.\quad-\frac{(R-1)(R-\bar{r})}{1-\bar{r}} \phi_{n}(1) b_{2}\left(1-e^{-s_{2} \tau} \cos \omega_{2} \tau\right)\right]\right\} \\
& \quad+\left(\frac{1-R}{1-\bar{r}}\right)^{2}\left(a_{1}-b_{1} e^{-s_{1} \tau} \cos \omega_{1} \tau\right)
\end{aligned}
$$

where the $q_{n}(\tau)$ 's are defined in (37). The associated $\xi_{n}(\tau)$ now is

$$
\xi_{n}(\tau)=\frac{-\gamma_{n} \dot{\psi}(\tau)+\bar{\gamma}_{n} \psi(\tau)}{1-\beta_{n} b_{2}\left(1-e^{-s_{2} \tau} \cos \omega_{2} \tau\right)} .
$$

To avoid numerical instability that occurred in computing $q_{n}(\tau),(37)$ is rewritten as

$$
q_{n}(\tau)=Q_{n}(\tau) q_{n}(0)+\int_{0}^{\tau} \xi_{n}(\varphi) \cdot \frac{Q_{n}(\tau)}{Q_{n}(\varphi)} d \varphi .
$$

Since the initial conditions cannot have effect on the steady-state response, we consider only the heat conduction in a hollow cylinder with constant initial temperature $\theta_{0}(X)=$ $\bar{\theta}_{0}$ as prescribed in the previous sections. The $q_{n}(0)$ 's are now computed as

$$
q_{n}(0)=\left[\xi_{J 1}-\frac{J_{0}\left(\lambda_{n} \bar{r}\right)}{Y_{0}\left(\lambda_{n} \bar{r}\right)}\right] \bar{\theta}_{0}-\gamma_{n} \psi(0) .
$$

For consistence in the temperature field, the constant $\bar{\theta}_{0}$ is taken as zero in the following examples.

In comparison with the literature, the example of constant Biot function is studied first. $\operatorname{Bi}(\tau)=1$ and time-dependent temperature function, $\psi(\tau)=1-e^{-\tau}$, are chosen in the case. In Table 1, we find that the convergence of the present solution is faster than that of Özisik [1]. The error of threeterm approximation in present study is less than $0.4 \%$; on the contrary, at least twenty-term approximation is required to get the same accuracy in Özisik's [1] cases.

In the case of time-dependent boundary condition and time-dependent heat transfer coefficient at both surfaces, we consider the time-dependent temperature function, $\psi(\tau)=$ $1-e^{-\tau} \cos \tau$, and the Biot function, $\operatorname{Bi}(\tau)=2-e^{-\tau}$. From Table 2, one can find that the error of three-term approximation is less than $0.4 \%$. Because of large values of $\operatorname{Bi}(\tau)$, the internal conductance of the hollow cylinder is small, whereas the heat transfer coefficient at the surface is large. In turn, the fact implies that the temperature distribution within the hollow cylinder is nonuniform. Therefore, we find that the larger the Biot function, that is, when $\tau$ approaches to 10 in Table 2, the more the iteration numbers.

Figure 2 depicts the temperature profiles along the radial of the hollow cylinder at different times, $\tau=0.1$ and $\tau=1$. We find that the temperature at $R=0.6$ is higher than the temperature at $R=1$ and the temperature profile decreases at the negative slope for every case. It is clear since the heat

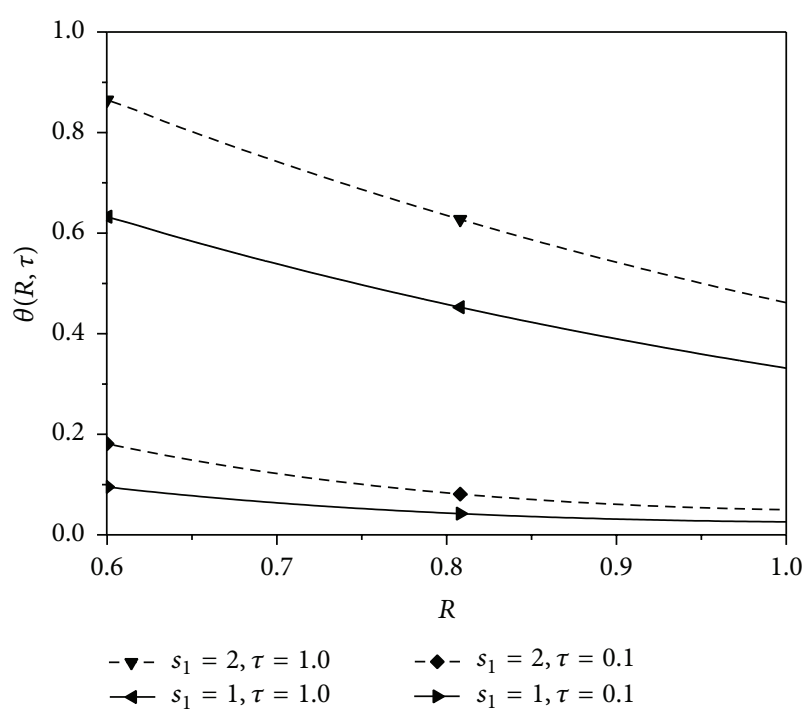

FIgURE 2: Temperature distribution and variation along the radial of the hollow cylinder with different parameter $s_{1}$ of temperature function $\psi(\tau),\left[\psi(\tau)=1-e^{-s_{1} \tau}, \operatorname{Bi}(\tau)=2-e^{-\tau}, \bar{\theta}_{0}=0\right]$.

TABLE 1: Temperatures of the hollow cylinder at outer surface and at various times $\left[\psi(\tau)=1-e^{-\tau}, \operatorname{Bi}(\tau)=1, \bar{\theta}_{0}=0\right]$.

\begin{tabular}{lcccccc}
\hline \multicolumn{4}{c}{} & \multicolumn{4}{c}{$\theta(1, \tau)$} \\
\multicolumn{2}{c}{2 terms } & \multicolumn{2}{c}{3 terms } & \multicolumn{2}{c}{20 terms } \\
& $\mathrm{A}$ & $\mathrm{B}$ & $\mathrm{A}$ & $\mathrm{B}$ & $\mathrm{A}$ & $\mathrm{B}$ \\
\hline 0.1 & 0.0263 & 0.0155 & 0.0261 & 0.0337 & 0.0262 & 0.0250 \\
0.5 & 0.2287 & 0.1841 & 0.2296 & 0.2610 & 0.2297 & 0.2248 \\
1 & 0.3981 & 0.3263 & 0.3998 & 0.4502 & 0.3998 & 0.3919 \\
5 & 0.6541 & 0.5416 & 0.6591 & 0.7364 & 0.6598 & 0.6449 \\
10 & 0.6578 & 0.5456 & 0.6675 & 0.7417 & 0.6690 & 0.6495 \\
\hline
\end{tabular}

A: present solution, (39); B: Özisik [1], (44).

TABLE 2: Temperatures of the hollow cylinder at outer surface and at various times $\left[\psi(\tau)=1-e^{-\tau} \cos \tau, \operatorname{Bi}(\tau)=2-e^{-\tau}, \bar{\theta}_{0}=0\right]$.

\begin{tabular}{lcccc}
\hline$\tau$ & \multicolumn{4}{c}{$\theta(1, \tau)$} \\
& 2 terms & 3 terms & 10 terms & 20 terms \\
\hline 0.1 & 0.0267 & 0.0265 & 0.0266 & 0.0266 \\
0.5 & 0.2398 & 0.2408 & 0.2408 & 0.2408 \\
1 & 0.4163 & 0.4185 & 0.4184 & 0.4185 \\
5 & 0.4900 & 0.4951 & 0.4951 & 0.4958 \\
10 & 0.4889 & 0.4987 & 0.4989 & 0.5001 \\
\hline
\end{tabular}

source comes to the hollow cylinder from inner surface $R=$ 0.6 , and the heat dissipates from $R=1$ to the surrounding environment.

Variable heat source versus variable Biot function is drawn to show the temperature variation of the hollow cylinder at $R=0.8$ and $R=1.0$ with respect to $\tau$ in Figures $3(\mathrm{a})$ and 3(b), respectively. Two cases of Biot function $\operatorname{Bi}(\tau)=$ $2-e^{-\tau}$ (solid lines) and $\operatorname{Bi}(\tau)=2-e^{-2 \tau}$ (dash lines) are considered. Due to the fact that the function $e^{-2 \tau}$ severely decays as time goes, therefore, in the same temperature 


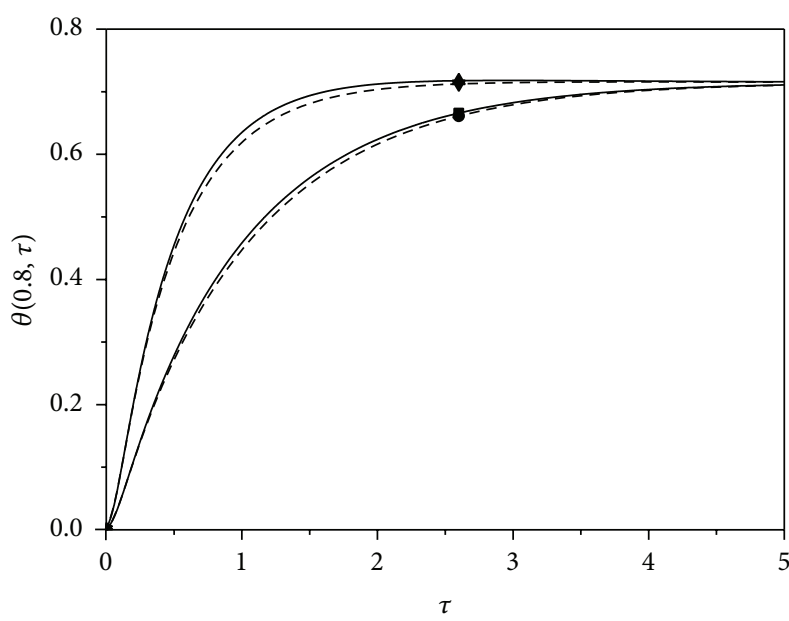

(a) At $R=0.8$

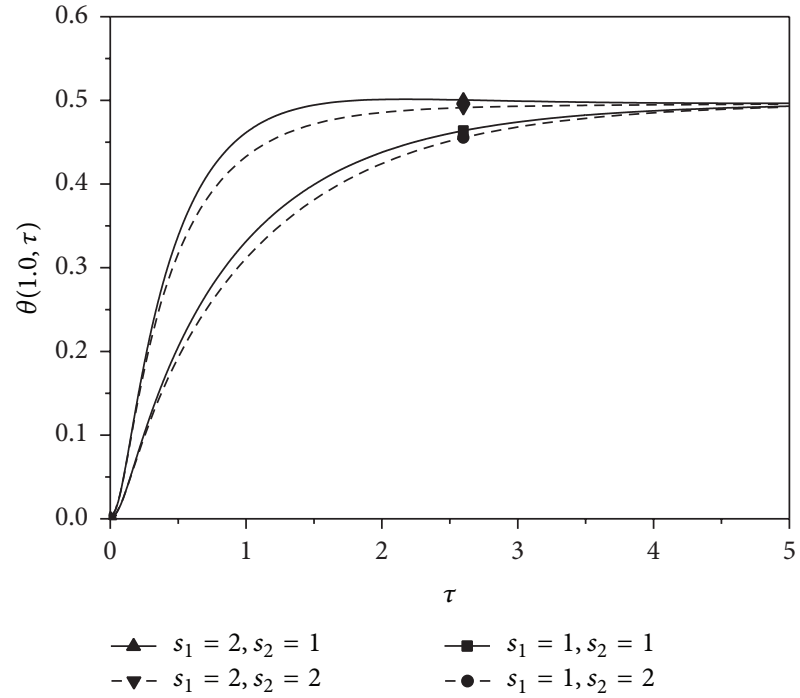

(b) At $R=1$

FIGURE 3: Influence of function parameters $s_{1}$ and $s_{2}$ on the temperatures of the hollow cylinder at middle and right surfaces, $\left[\psi(\tau)=1-e^{-s_{1} \tau}\right.$, $\left.\operatorname{Bi}(\tau)=2-e^{-s_{2} \tau}, \bar{\theta}_{0}=0\right]$

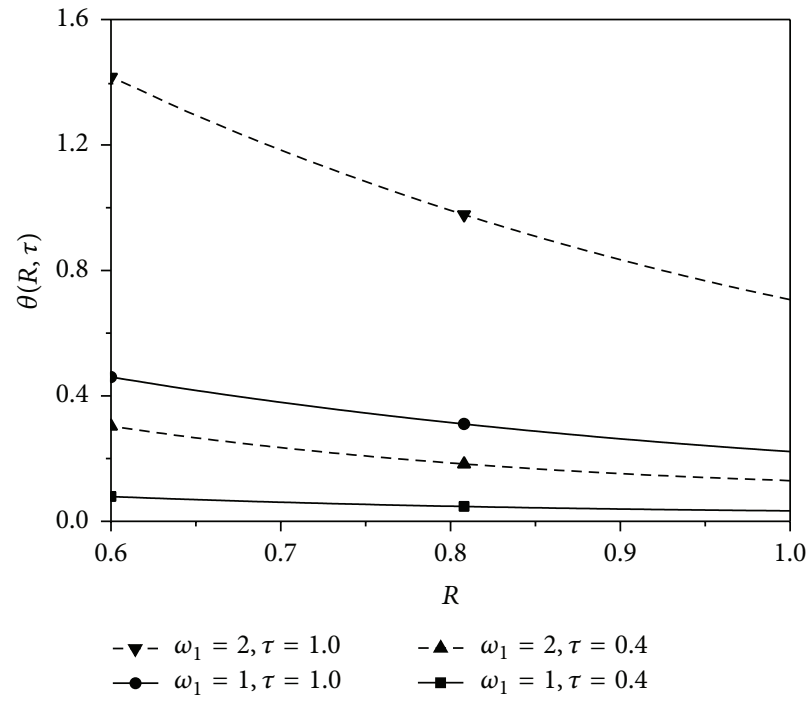

FIGURE 4: Temperature distribution and variation along the radial of the hollow cylinder with different parameter $\omega_{1}$ of temperature function $\psi(\tau),\left[\psi(\tau)=1-\cos \omega_{1} \tau, \operatorname{Bi}(\tau)=2-e^{-\tau}, \bar{\theta}_{0}=0\right]$.

function $\psi(\tau)$ the temperature in $\operatorname{Bi}(\tau)=2-e^{-2 \tau}$ is less than that in $\operatorname{Bi}(\tau)=2-e^{-\tau}$ as $\tau$ proceeds. That is to say, more heat will be dissipated into the surrounding environment for $\operatorname{Bi}(\tau)=2-e^{-2 \tau}$ as $\tau$ goes.

Figure 4 depicts the effect of the parameter $\omega_{1}$ of temperature function $\psi(\tau)$ upon the temperature variation of the hollow cylinder. It is found that, in the same temperature function $\psi(\tau)$, the temperature for $\tau=0.4$ is less than that for $\tau=1.0$. Besides, as $\tau$ increases from 0.4 to 1.0 , the difference between temperatures at $\omega_{1}=1$ and at $\omega_{1}=2$ becomes significant.
Periodical heat source versus time-varying Biot function is drawn to show the temperature variation of the hollow cylinder at $R=0.8$ and $R=1.0$ with respect to $\tau$ in Figures $5(\mathrm{a})$ and 5(b), respectively. Two cases of heat source $\psi(\tau)=$ $1-\cos \tau$ (solid lines) and $\psi(\tau)=1-\cos 2 \tau$ (dash lines) are considered. At the same $\tau$, the temperature of $\operatorname{Bi}(\tau)=2-e^{-\tau}$ is less than that of $\operatorname{Bi}(\tau)=2-e^{-0.1 \tau}$ for constant $\psi(\tau)$. The reason is that more heat has been dissipated into the surrounding environment at the case of $\operatorname{Bi}(\tau)=2-e^{-\tau}$. It can be observed that as $\tau$ proceeds, in the beginning, the temperatures are nonsensitive with $\omega_{1}$ parameters, as shown in Figure 5. 


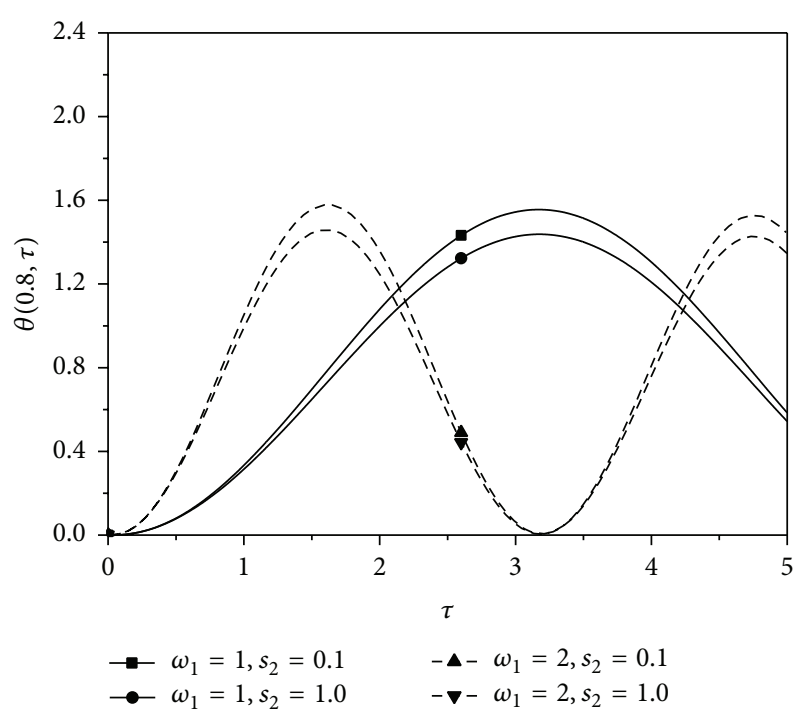

(a) At $R=0.8$

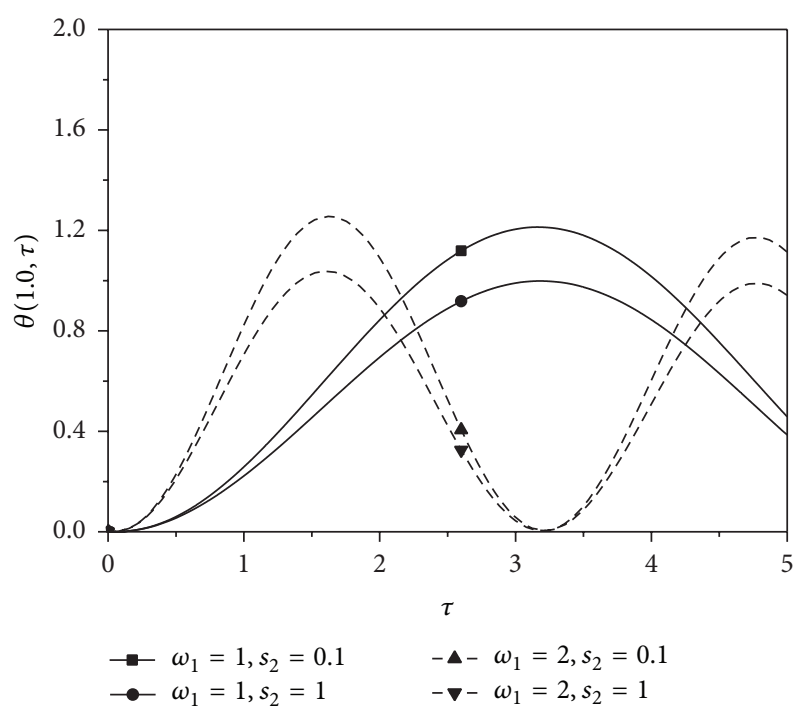

(b) At $R=1$

Figure 5: Influence of $\omega_{1}$ parameter and $s_{2}$ parameter on the temperature variation of the hollow cylinder at middle and right surfaces, $\left[\psi(\tau)=1-\cos \omega_{1} \tau, \operatorname{Bi}(\tau)=2-e^{-s_{2} \tau}, \bar{\theta}_{0}=0\right]$.

\section{Conclusion}

An analytical solution for the heat conduction in a hollow cylinder with time-dependent boundary conditions of different kinds at both surfaces was developed for the first time. The surface is subject to a time-dependent temperature field at inner surface, whereas the heat is dissipated by timedependent convection from outer surface into a surrounding environment at zero temperature. The methodology is an extension of the shifting function method and the present results are identical to those in the literature when constant Biot function is considered. Since the methodology does not use integral transform, it has a proven result. The proposed method can also be easily extended to various heat conduction problems of hollow cylinders with timedependent boundary conditions of different kinds at both surfaces.

\section{Nomenclatures}

$\begin{array}{ll}a, b: & \text { Inner and outer radii (m) } \\ a_{1}, b_{1}, a_{2}, b_{2}: & \text { Arbitrary constants used to express } \\ \text { Bi: } & \text { temperature and Biot functions } \\ d_{1}, d_{2}: & \text { Biot function } \\ f_{1}, f_{2}: & \text { Auxiliary functions } \\ F: & \text { Auxiliary time functions } \\ g_{1}, g_{2}: & \text { Biot function minus a constant } \\ h: & \text { Shifting functions } \\ H: & \text { Time-dependent heat transfer coefficient } \\ & \text { at outer surface }\left(\mathrm{W} \cdot \mathrm{m}^{-2} \cdot \mathrm{K}^{-1}\right) \\ J_{0}: & \text { Variable temperature function at inner } \\ & \text { surface }(\mathrm{K}) \\ k: & \text { Bessel function of order zero of the first } \\ N_{n}: & \text { kind } \\ q_{n}: & \text { Thermal conductivity }\left(\mathrm{W} \cdot \mathrm{m}^{-1} \cdot \mathrm{K}^{-1}\right) \\ r: & \text { Norm of try functions } \\ \bar{r}: & \text { Time-dependent generalized coordinates } \\ R: & \text { Space variable }(\mathrm{m}) \\ s_{1}, s_{2}: & \text { Ratio of inner radius over outer radius } \\ t: & \text { Dimensionless radius } \\ T: & \text { Parameters used to express temperature } \\ T_{r}: & \text { and Biot functions } \\ T_{0}: & \text { Time variable }(\text { sec) } \\ v: & \text { Temperature }(\mathrm{K}) \\ Y_{0}: & \text { Constant reference temperature }(\mathrm{K}) \\ & \text { Initial temperature }(\mathrm{K}) \\ & \text { Auxiliary function } \\ & \text { Bessel function of order zero of the } \\ & \text { second kind. }\end{array}$

Greek Symbols

$\alpha: \quad$ Thermal diffusivity $\left(\mathrm{m}^{2} \cdot \mathrm{s}^{-1}\right)$

$\beta_{n}, \bar{\beta}_{n}:$ Auxiliary functions

$\delta: \quad$ Initial value of Biot function

$\phi_{n}: \quad$ Eigenfunctions

$\varphi$ : $\quad$ Auxiliary integration variable

$\gamma_{n}, \bar{\gamma}_{n}:$ Auxiliary functions

$\lambda_{n}: \quad$ Eigenvalues

$\theta: \quad$ Dimensionless temperature

$\theta_{0}$ : Dimensionless initial temperature

$\tau: \quad$ Dimensionless time variable

$\omega_{1}, \omega_{2}$ : Parameters for temperature and Biot functions

$\xi_{n}: \quad$ Auxiliary function

$\xi_{W i}: \quad$ Auxiliary functions to express integration terms of Bessel functions

$\psi$ : Dimensionless time-dependent temperature

function

$\zeta$ : $\quad$ Auxiliary integration variable.

\section{Subscripts}

$0,1,2, m, n, J, Y$ : Indices. 


\section{Conflict of Interests}

The authors declare that there is no conflict of interests regarding the publication of this paper.

\section{Acknowledgment}

It is gratefully acknowledged that this work was supported by the National Science Council of Taiwan, under Grants NSC 103-2221-E-006-048 and NSC 95-2221-E-344-001.

\section{References}

[1] M. N. Özisik, "Heat conduction in the cylindrical coordinate system," in Boundary Value Problems of Heat Conduction, chapter 3, pp. 125-193, International Textbook Company, Scranton, Pa, USA, 1st edition, 1968.

[2] V. V. Ivanov and V. V. Salomatov, "On the calculation of the temperature field in solids with variable heat-transfer coefficients," Journal of Engineering Physics and Thermophysics, vol. 9, no. 1, pp. 63-64, 1965.

[3] V. V. Ivanov and V. V. Salomatov, "Unsteady temperature field in solid bodies with variable heat transfer coefficient," Journal of Engineering Physics and Thermophysics, vol. 11, no. 2, pp. 151152, 1966.

[4] Y. S. Postol'nik, "One-dimensional convective heating with a time-dependent heat-transfer coefficient," Journal of Engineering Physics and Thermophysics, vol. 18, no. 2, pp. 316-322, 1970.

[5] V. N. Kozlov, "Solution of heat-conduction problem with variable heat-exchange coefficient," Journal of Engineering Physics and Thermophysics, vol. 18, no. 1, pp. 133-138, 1970.

[6] N. M. Becker, R. L. Bivins, Y. C. Hsu, H. D. Murphy, A. B. White Jr., and G. M. Wing, "Heat diffusion with timedependent convective boundary condition," International Journal for Numerical Methods in Engineering, vol. 19, no. 12, pp. 1871-1880, 1983.

[7] H. T. Chen, S. L. Sun, H. C. Huang, and S. Y. Lee, "Analytic closed solution for the heat conduction with time dependent heat convection coefficient at one boundary," Computer Modeling in Engineering \& Sciences, vol. 59, no. 2, pp. 107-126, 2010.

[8] O. I. Yatskiv, R. M. Shvets', and B. Y. Bobyk, “Thermostressed state of a cylinder with thin near-surface layer having timedependent thermophysical properties," Journal of Mathematical Sciences, vol. 187, no. 5, pp. 647-666, 2012.

[9] Z. J. Holy, "Temperature and stresses in reactor fuel elements due to time- and space-dependent heat-transfer coefficients," Nuclear Engineering and Design, vol. 18, no. 1, pp. 145-197, 1972.

[10] M. N. Özisik and R. L. Murray, "On the solution of linear diffusion problems with variable boundary condition parameters," ASME Journal of Heat Transfer, vol. 96, no. 1, pp. 48-51, 1974.

[11] M. D. Mikhailov, "On the solution of the heat equation with time dependent coefficient," International Journal of Heat and Mass Transfer, vol. 18, no. 2, pp. 344-345, 1975.

[12] R. M. Cotta and C. A. C. Santos, "Nonsteady diffusion with variable coefficients in the boundary conditions," Journal of Engineering Physics and Thermophysics, vol. 61, no. 5, pp. 14111418, 1991.

[13] R. J. Moitsheki, “Transient heat diffusion with temperaturedependent conductivity and time-dependent heat transfer coefficient," Mathematical Problems in Engineering, vol. 2008, Article ID 347568, 9 pages, 2008.
[14] S. Chantasiriwan, "Inverse heat conduction problem of determining time-dependent heat transfer coefficient," International Journal of Heat and Mass Transfer, vol. 42, no. 23, pp. 4275-4285, 1999.

[15] J. Su and G. F. Hewitt, "Inverse heat conduction problem of estimating time-varying heat transfer coefficient," Numerical Heat Transfer, Part A: Applications, vol. 45, no. 8, pp. 777-789, 2004.

[16] J. Zueco, F. Alhama, and C. F. G. Fernández, "Inverse problem of estimating time-dependent heat transfer coefficient with the network simulation method," Communications in Numerical Methods in Engineering, vol. 21, no. 1, pp. 39-48, 2005.

[17] D. Słota, "Using genetic algorithms for the determination of an heat transfer coefficient in three-phase inverse Stefan problem," International Communications in Heat and Mass Transfer, vol. 35, no. 2, pp. 149-156, 2008.

[18] H.-T. Chen and X.-Y. Wu, "Investigation of heat transfer coefficient in two-dimensional transient inverse heat conduction problems using the hybrid inverse scheme," International Journal for Numerical Methods in Engineering, vol. 73, no. 1, pp. 107-122, 2008.

[19] T. T. M. Onyango, D. B. Ingham, D. Lesnic, and M. Slodička, "Determination of a time-dependent heat transfer coefficient from non-standard boundary measurements," Mathematics and Computers in Simulation, vol. 79, no. 5, pp. 1577-1584, 2009.

[20] J. Sladek, V. Sladek, P. H. Wen, and Y. C. Hon, "The inverse problem of determining heat transfer coefficients by the meshless local Petrov-Galerkin method," Computer Modeling in Engineering \& Sciences, vol. 48, no. 2, pp. 191-218, 2009.

[21] S. Y. Lee and S. M. Lin, "Dynamic analysis of nonuniform beams with time-dependent elastic boundary conditions," ASME Journal of Applied Mechanics, vol. 63, no. 2, pp. 474-478, 1996.

[22] S. Y. Lee, S. Y. Lu, Y. T. Liu, and H. C. Huang, "Exact large deflection of Timoshenko beams with nonlinear boundary conditions," Computer Modeling in Engineering \& Sciences, vol. 33, no. 3, pp. 293-312, 2008. 


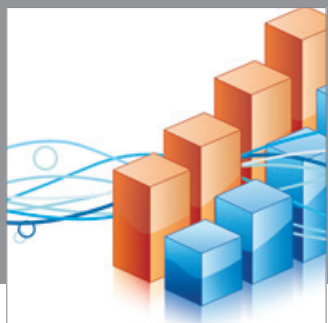

Advances in

Operations Research

mansans

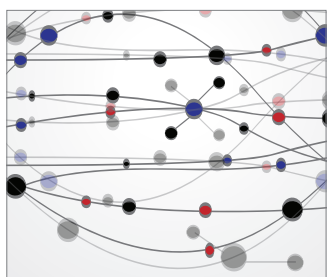

The Scientific World Journal
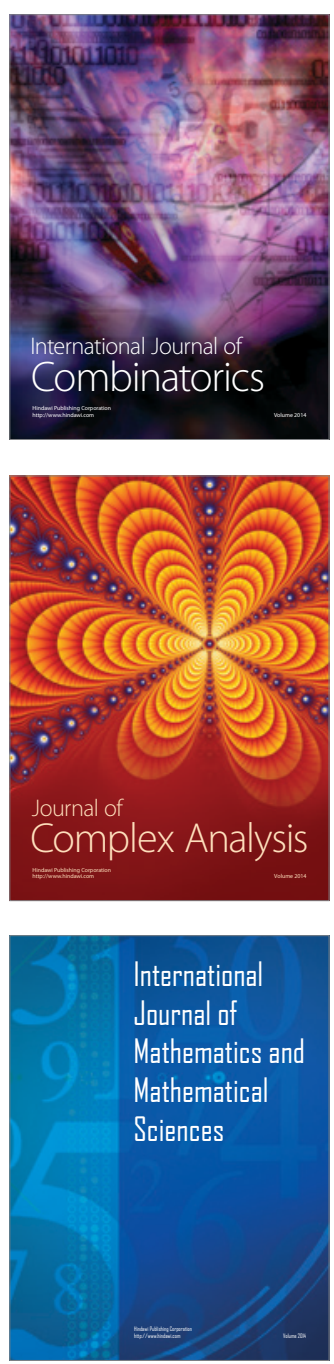
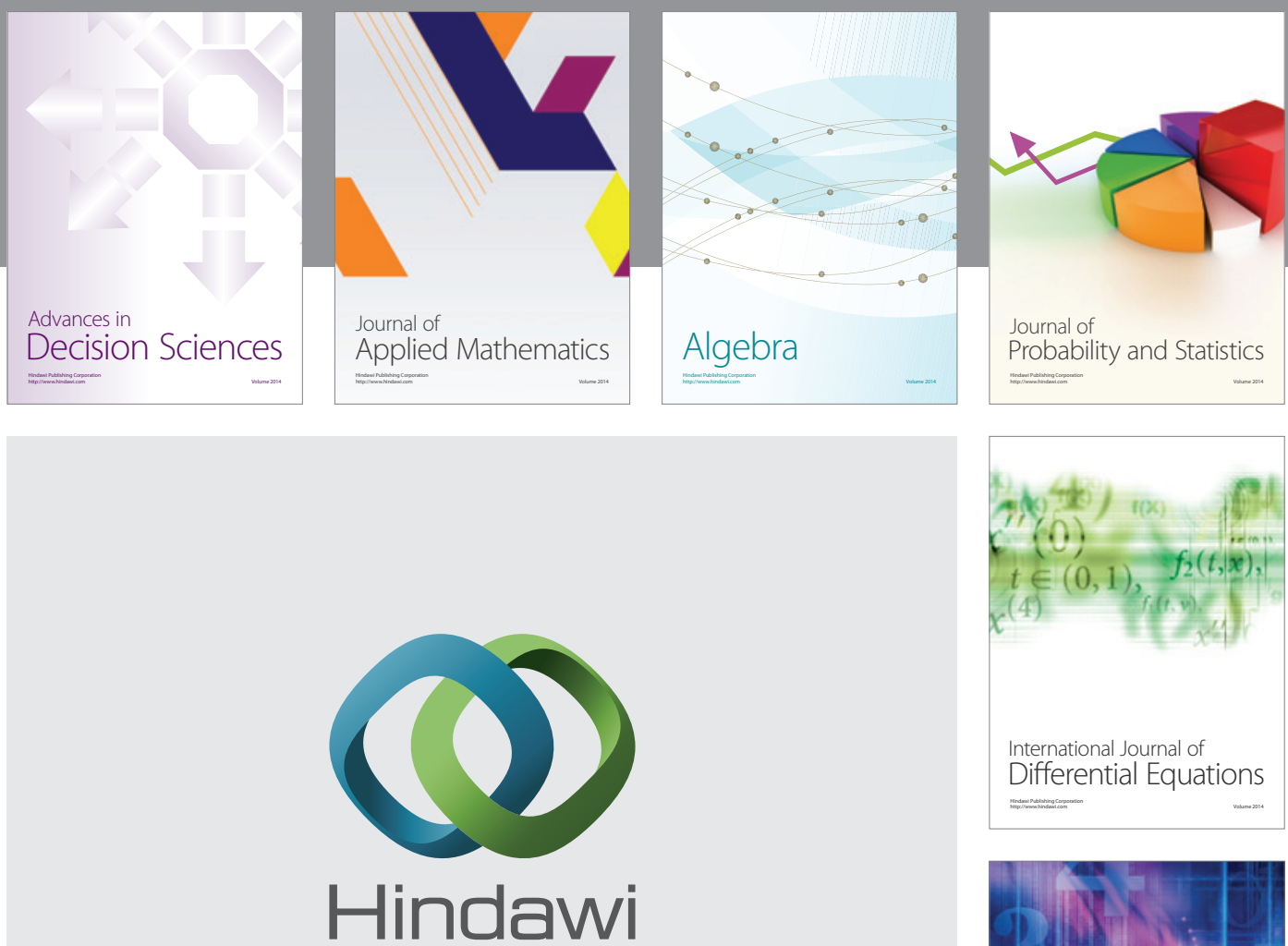

Submit your manuscripts at http://www.hindawi.com
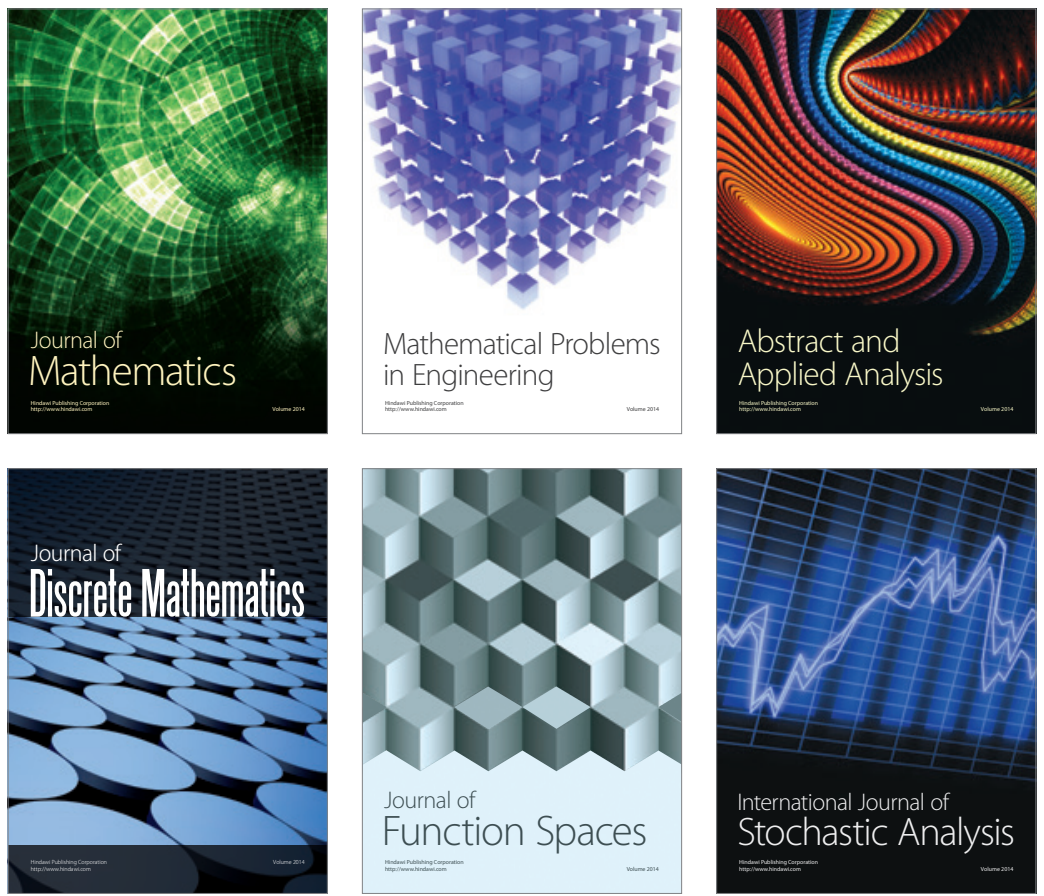

Journal of

Function Spaces

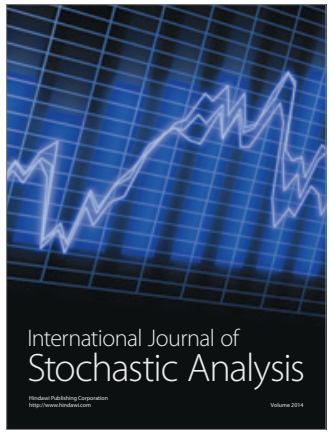

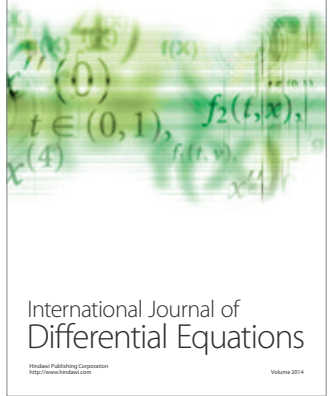
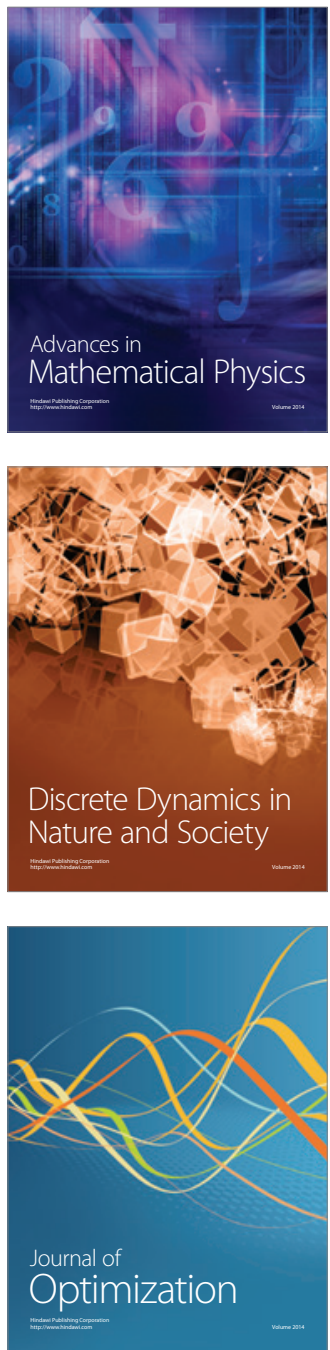